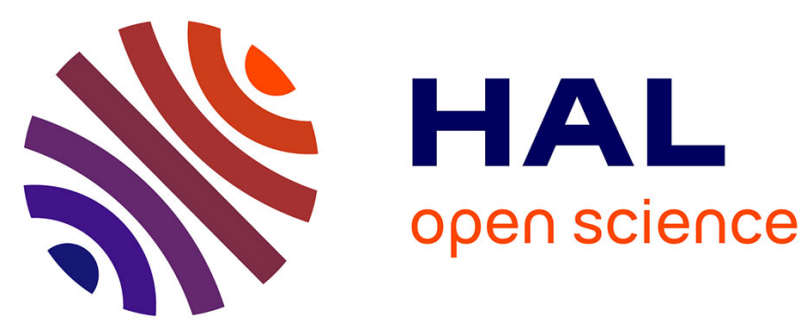

\title{
Comparison of Biomarkers in Transgenic Alzheimer Rats Using Multi-shell Diffusion MRI
}

Rutger H.J. Fick, Madelaine Daianu, Marco Pizzolato, Demian Wassermann, Russel E. Jacobs, Paul M. Thompson, Terrence Town, Rachid Deriche

\section{- To cite this version:}

Rutger H.J. Fick, Madelaine Daianu, Marco Pizzolato, Demian Wassermann, Russel E. Jacobs, et al.. Comparison of Biomarkers in Transgenic Alzheimer Rats Using Multi-shell Diffusion MRI. MICCAI 2016 Workshop on Computational Diffusion MRI (CDMRI'16), Oct 2016, Athènes, Greece. hal01354981

\section{HAL Id: hal-01354981 \\ https://hal.science/hal-01354981}

Submitted on 21 Aug 2016

HAL is a multi-disciplinary open access archive for the deposit and dissemination of scientific research documents, whether they are published or not. The documents may come from teaching and research institutions in France or abroad, or from public or private research centers.
L'archive ouverte pluridisciplinaire HAL, est destinée au dépôt et à la diffusion de documents scientifiques de niveau recherche, publiés ou non, émanant des établissements d'enseignement et de recherche français ou étrangers, des laboratoires publics ou privés. 


\title{
Comparison of Biomarkers in Transgenic Alzheimer Rats Using Multi-shell Diffusion MRI
}

\author{
Rutger H.J. Fick ${ }^{1}$, Madelaine Daianu ${ }^{2}$, Marco Pizzolato ${ }^{1}$, \\ Demian Wassermann ${ }^{1}$, Russell E. Jacobs ${ }^{3}$, \\ Paul M. Thompson ${ }^{2}$, Terrence Town ${ }^{4}$, and Rachid Deriche ${ }^{1}$ \\ ${ }^{1}$ Université Côte d'Azur, Inria, France \\ ${ }^{2}$ Imaging Genetics Center, Mark \& Mary Stevens Neuroimaging \& Informatics \\ Institute, University of Southern California, Marina del Rey, CA, USA \\ ${ }^{3}$ Division of Biology and Biological Engineering, Beckman Institute, California \\ Institute of Technology, Pasadena, CA, USA \\ ${ }^{4}$ Department of Physiology \& Biophysics, Zilkha Neurogenetic Institute, University \\ of Southern California, Los Angeles, CA, USA
}

\begin{abstract}
In this study, we assessed the evolution of diffusion MRI (dMRI) derived markers from different white matter models as progressive neurodegeneration occurs in transgenic Alzheimer rats (TgF344$\mathrm{AD})$ at 10, 15 and 24 months. We compared biomarkers reconstructed from Diffusion Tensor Imaging (DTI), Neurite Orientation Dispersion and Density Imaging (NODDI) and Mean Apparent Propagator (MAP)MRI in the hippocampus, cingulate cortex and corpus callosum using multi-shell dMRI. We found that NODDI's dispersion and MAP-MRI's anisotropy markers consistently changed over time, possibly indicating that these measures are sensitive to age-dependent neuronal demise due to amyloid accumulation. Conversely, we found that DTI's mean diffusivity, NODDI's isotropic volume fraction and MAP-MRI's restrictionrelated metrics all followed a two-step progression from 10 to 15 months, and from 15 to 24 months. This two-step pattern might be linked with a neuroinflammatory response that may be occuring prior to, or during microstructural breakdown. Using our approach, we are able to provide - for the first time - preliminary and valuable insight on relevant biomarkers that may directly describe the underlying pathophysiology in Alzheimer's disease.
\end{abstract}

\section{Introduction}

Diffusion MRI (dMRI) allows us to non-invasively study microstructural changes caused by neuropathology. Among these pathologies, gaining understanding of Alzheimer's disease (AD) is of particular importance, affecting over one in nine people age 65 and above in the U.S. alone [1]. Traditionally, dMRI studies have used Diffusion Tensor Imaging (DTI) [2] to model the grey and white matter structure abnormalities in AD patients. Only recently, more complex white matter models like Neurite Orientation Dispersion and Density Imaging (NODDI) [3] have been explored to classify $\mathrm{AD}$, and have shown greater discriminative power 
than DTI [4]. This reinforces the importance of exploring white matter models that provide more detailed microstructural information than DTI.

In human studies, it is hard to relate dMRI derived metrics to corresponding microstructural changes for lack of histological validation. As a solution, animal models provide a way to gain understanding on the underlying pathophysiology of AD by allowing dMRI in addition to histological measurements. Mouse models of human tauopathy ( $\mathrm{rTg} 4510$ ) have been previously studied at various time points using DTI $[5,6]$, and at a single time point comparing DTI with NODDI metrics [7]. In this latter study, NODDI derived metrics once again appeared more discriminative than those derived from DTI. Further efforts focusing on multi-shell dMRI analysis of transgenic Alzheimer rats (TgF344-AD) have shown that dMRI measurements at higher gradient strengths aid the classication of AD-like pathology [8]. However, only anisotropy measures of DTI and hybrid diffusion imaging (HYDI) [9] were explored.

In this study, we compare the evolution of dMRI-derived markers from different white matter models as progressive neurodegeneration occurs in transgenic Alzheimer rats (TgF344-AD). In particular, we study the patterns of alteration across three time points in the hippocampus, cingulate cortex and corpus callosum - areas known to be affected in AD. The two grey matter areas were previously shown to manifest age-dependent cerebral amyloidosis that precedes tauopathy, gliosis and apoptotic loss of neurons [10], making these cortical regions extremely relevant for understanding the underlying mechanisms in $\mathrm{AD}$. We compare biomarkers derived from DTI, NODDI and Mean Apparent Propagator (MAP)-MRI [11] using multi-shell data. To the best of our knowledge, this is the first study that investigates multi-shell biomarkers at different time points in $\mathrm{AD}$ animal models.

The paper is structured as follows: we first describe the diffusion MRI data and the metrics we derive in Section 2. We provide the results in section 3 and discuss them in section 4 . We finally provide our conclusions in section 5 .

\section{Materials and Methods}

In this section, we first detail the diffusion MRI data acquisition, preprocessing and region of interest selection of the $\mathrm{AD}$ rats. We then give a brief overview of the methods we use and their metrics of interest. We detail the fractional anisotropy (FA) and mean diffusivity (MD) of classical DTI, the orientation dispersion index (ODI), neurite density index (NDI) and isotropic volume fraction (IsoVF) of the multi-compartment NODDI model, and finally the formulation of several q-space indices of the MAP-MRI functional basis. We estimated the DTI and MAP-MRI metrics using the diffusion imaging in python (dipy) open source software [12] and the NODDI metrics using the NODDI toolbox [3].

\subsection{Processing of Transgenic Alzheimer Rat Data Sets}

We use multi-shell dMRI data of three ex-vivo transgenic Alzheimer rats (line TgF344-AD) [10], also previously analyzed by Daianu et al. [8]. The rats were 

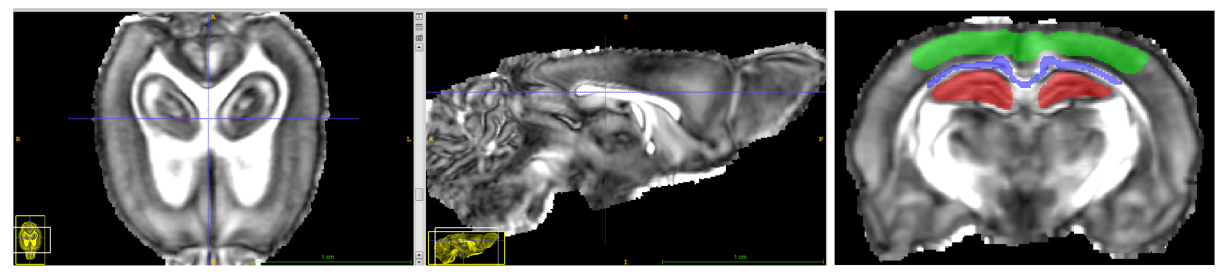

Fig. 1. Regions of interest for biomarker estimation on the registered FA map of rat 1. We mark the cingulate cortex (green), corpus callosum (blue) and hippocampus (red).

euthanized at 10, 15 and 24 months, fixed brains were prepared as described in [8], and scanned using a 7 Tesla Bruker Biospin MRI scanner at California Institute of Technology. A high-resolution fast low angle magnetic shot (FLASH) anatomical image with a mix of T1 and T2 weighting $(375 \times 224 \times 160$ matrix; voxel size: $0.08 \times 0.08 \times 0.08 \mathrm{~mm}^{3}$ ) was used. The diffusion MRI data were sampled on 5 shells with b-values $\{1000,3000,4000,8000,12000\} \mathrm{s} / \mathrm{mm}^{2}$, all with the same 60 directions and 5 b0 measurements. Other parameters were $\delta / \Delta=11 / 16 \mathrm{~ms}$ and $T E / T R=34 / 500 \mathrm{~ms}$. The voxel dimensions were $0.15 \times 0.15 \times 0.25 \mathrm{~mm}^{3}$.

During preprocessing, extra-cerebral tissue was removed using the skullstripping Brain Extraction Tool from BrainSuite (http://brainsuite.org/), for both the anatomical images and the DWIs. We corrected for eddy current distortions using the eddy correct FSL tool (www.fmrib.ox.ac.uk/fsl) for which a gradient table was calculated to account for the distortions. As an image processing step, DWIs were up-sampled to the resolution of the anatomical images (with isotropic voxels) using FSLs flirt function with 9 degrees of freedom; the gradient direction tables were rotated accordingly after each linear registration. For our study, we draw regions of interest (ROIs) in the cingulate cortex, hippocampus and corpus callosum as shown in Figure 1.

\subsection{DTI Metrics}

The classical DTI model [2] assumes that the measured diffusion signal belongs to the set of Gaussian distributions. While DTI has well-known limitations with respect to the modeling of crossing tissue configurations and restricted diffusion, its derived metrics $\mathrm{FA}$ and $\mathrm{MD}$ have been found useful to classify $\mathrm{AD}$ patients [4]. Using signal attenuation $E(b)=S(b) / S(0)$, the DTI model describes the diffusion signal as $E(b)=\exp \left(-b \mathbf{g}^{\mathrm{T}} \mathbf{D g}\right)$ with $\mathbf{D}$ a $3 \times 3$ symmetric positive-definite matrix and $\mathbf{g}$ the gradient direction. Estimating the eigenvalues of $\mathbf{D}$ as $\left\{\lambda_{1}, \lambda_{2}, \lambda_{3}\right\}$ the FA and MD are given as

$$
F A=\sqrt{\frac{1}{2}} \frac{\sqrt{\left(\lambda_{1}-\lambda_{2}\right)^{2}+\left(\lambda_{2}-\lambda_{3}\right)^{2}+\left(\lambda_{3}-\lambda_{1}\right)^{2}}}{\sqrt{\lambda_{1}^{2}+\lambda_{2}^{2}+\lambda_{3}^{2}}} \quad M D=\frac{\lambda_{1}+\lambda_{2}+\lambda_{3}}{3}
$$

In accordance with DTI's Gaussian diffusion assumption, we only use the b0 and $b=1000 \mathrm{~s} / \mathrm{mm}^{2}$ data when fitting DTI. The FA and MD in our slice of interest are shown in Figure 2. 


\subsection{NODDI Metrics}

The more advanced multi-compartment NODDI model [3] separates the signal contribution of different tissues by fitting a combination of intra-cellular, extracellular and free-water models.

$$
E=\left(1-\nu_{i s o}\right)\left(\nu_{i c} E_{i c}(O D I)+\left(1-\nu_{i c}\right) * E_{e c}\right)+\nu_{i s o} E_{i s o}
$$

The intra-cellular signal $E_{i c}$ is modeled as a set of dispersed sticks, i.e., cylinders of zero radius, to capture the highly restricted nature of diffusion perpendicular to neurites and unhindered diffusion along them. The amount of dispersion is given by the orientation dispersion index (ODI), which is defined by a Watson distribution. The extra-cellular signal $E_{e c}$ is described as a dispersed mixture of Gaussian anisotropic diffusion, and an isotropic Gaussian compartment $E_{\text {iso }}$ represents free diffusion. Similarly as in [7], we study the ODI, the neurite density index $N D I=\left(1-\nu_{i s o}\right) \nu_{i c}$ and the isotropic volume fraction $I s o V F=\nu_{i s o}$.

In accordance with NODDI's recommended acquisition scheme [3], we fit NODDI only using the b0 and $b=\{1000,3000\} \mathrm{s} / \mathrm{mm}^{2}$ data. Furthermore, as water diffusivity changes in ex-vivo tissue, we set the intra-cellular and isotropic diffusivity to $0.6 \times 10^{-9} \mathrm{~m}^{2} \mathrm{~s}^{-1}$ and $2.0 \times 10^{-9} \mathrm{~m}^{2} \mathrm{~s}^{-1}$ [13]. An illustration of the ODI, NDI and IsoVF can be seen in Figure 2.

\subsection{MAP-MRI Metrics}

The MAP-MRI approach [11] uses a functional basis to represent the 3D diffusion signal with as little assumptions as possible. It then analytically reconstructs the $3 \mathrm{D}$ diffusion propagator by only assuming the short gradient pulse approximation $(\delta \approx 0)$. In this way, it accurately estimates the diffusion propagator in the presence of both non-Gaussian diffusion and crossing tissue configurations.

MAP-MRI represents the discretely measured signal attenuation $E(\mathbf{q})$ using a set of continuous orthogonal basis functions representing the space $\hat{E}(\mathbf{q} ; \mathbf{c})$, where the signal is now represented in terms of basis coefficients $\mathbf{c}$ and the qspace wave vector $\mathbf{q}=|\mathbf{q}| \mathbf{g}$ with $\mathbf{g}$ the gradient direction is related to the b-value as $|\mathbf{q}|=\sqrt{b /(\Delta-\delta / 3)} / 2 \pi$. Without going into the formulation of MAP-MRI's basis functions, we detail the estimation of basis coefficients $\mathbf{c}$ in Eq. (3). In short, we regularize the fitting of $\mathbf{c}$ such that $\hat{E}(\mathbf{q} ; \mathbf{c})$ smoothly interpolates between the measured q-space points by using Laplacian regularization [14], where regularization weight $\lambda$ is set using voxel-wise generalized cross-validation. We also constrain the estimated diffusion Propagator $\hat{P}(\mathbf{R} ; \mathbf{c})$ to be positive using quadratic programming [11].

$$
\begin{aligned}
& \operatorname{argmin}_{\mathbf{c}} \overbrace{\int_{\mathbb{R}^{3}}[E(\mathbf{q})-\hat{E}(\mathbf{q} ; \mathbf{c})]^{2} d \mathbf{q}}^{\text {Data Fidelity }}+\overbrace{\lambda \int_{\mathbb{R}^{3}}\left[\nabla^{2} \hat{E}(\mathbf{q} ; \mathbf{c})\right]^{2} d \mathbf{q}}^{\text {Smoothness }} \\
& \text { subject to } \hat{P}(\mathbf{R} ; \mathbf{c})>0 \quad \text { with } \hat{P}(\mathbf{R} ; \mathbf{c})=\operatorname{IFT}(\hat{E}(\mathbf{q} ; \mathbf{c}))
\end{aligned}
$$




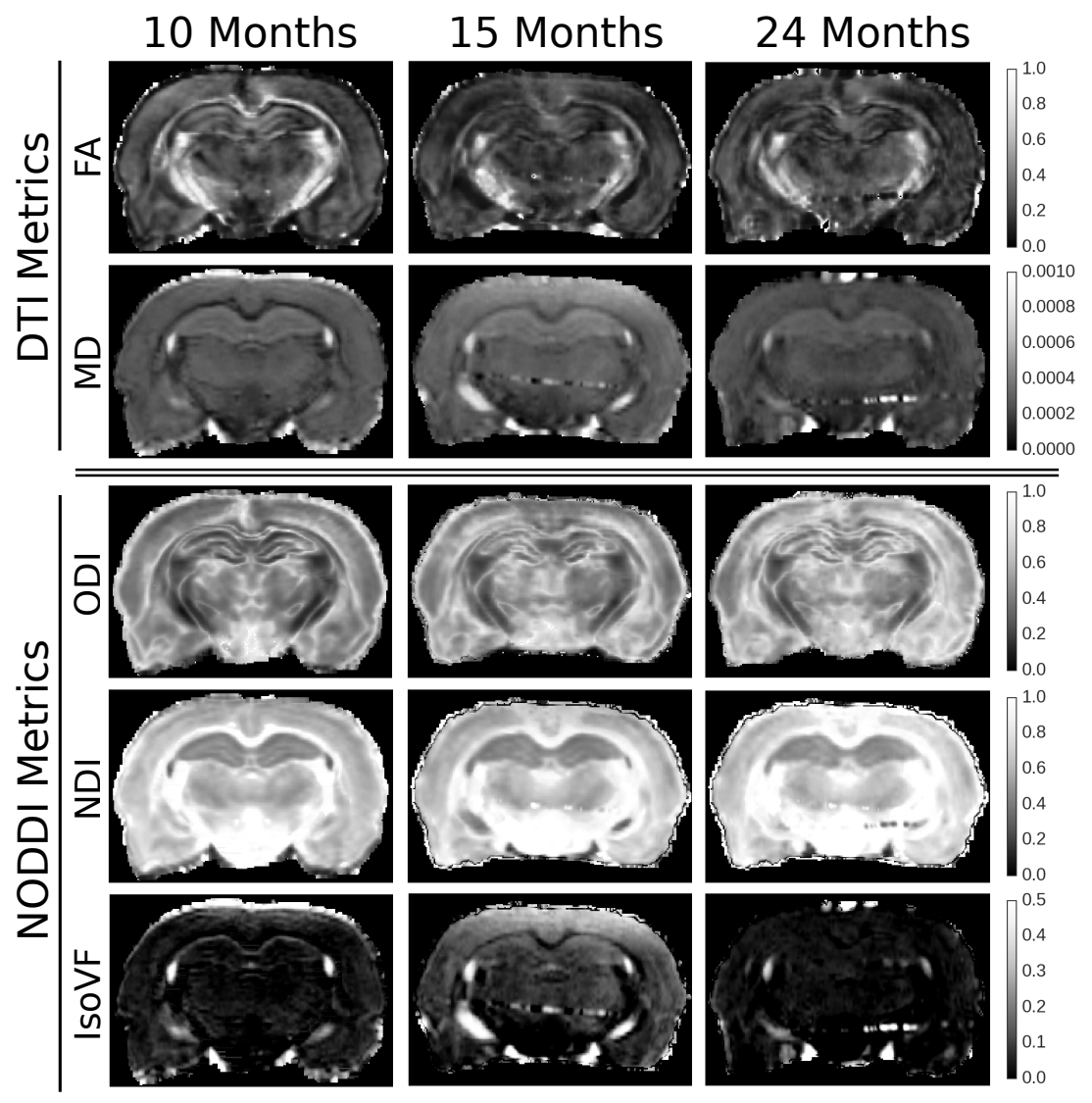

Fig. 2. Illustrations of a DTI and NODDI metrics in the same coronal slice for the three time points.

Once $\mathbf{c}$ is known, the MAP-MRI basis simultaneously represents the 3D dMRI signal and 3D diffusion propagator. We estimate the q-space indices Return-ToOrigin, Return-To-Axis and Return-To-Plane Probability (RTOP, RTAP and RTPP), which in theory are related to the volume, surface and length of a cylindrical pore [11]. We also estimate the non-Gaussianity (NG), which describes the ratio between the Gaussian and non-Gaussian volume of the signal. Finally we estimate the propagator anisotropy (PA), which is a normalized metric that describes the anisotropy of the 3D diffusion propagator. As MAP-MRI is designed to represent the entire 3D diffusion signal, we estimate all metrics using the entire 5 shell data up to a b-value of $12000 \mathrm{~s} / \mathrm{mm}^{2}$, using a radial order of 6 , resulting in 50 estimated coefficients. We illustrate these metrics in Figure 3. 


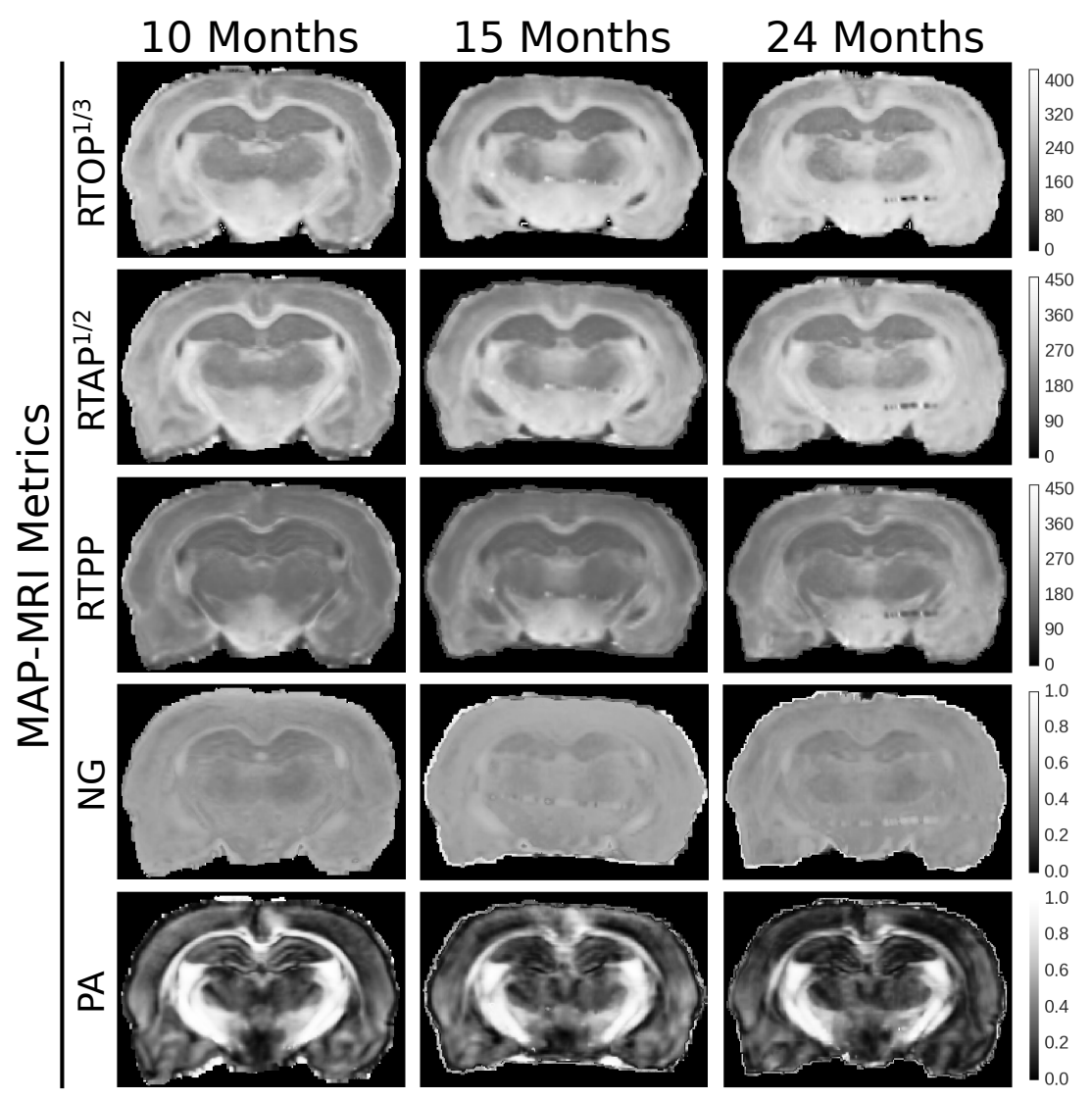

Fig. 3. Illustrations of MAP-MRI's q-space indices in the same coronal slice for the three time points. To visualize RTOP, RTAP and RTPP in the same unit $\left(\mathrm{mm}^{-1}\right)$ we show the cubed root of RTOP and squared root of RTAP.

\section{Results}

In Figure 4 we show the evolution of the mean with 0.5 standard deviation of all dMRI-derived metrics in the ROIs shown in Figure 1. We use the same colors for the hippocampus (red), corpus callosum (blue) and cingulate cortex (green). The only metric that consistently increases over time is NODDI's ODI and consistently decreases is MAP-MRI's PA, with the exception of the cortex. It is also apparent that FA, NDI, RTOP, RTAP and RTPP follow a different, 2-step pattern, first decreasing and then slightly increasing. Inversely, for MD, IsoVF and NG we first find an increase and then a decrease. We provide the raw data values in Table 1 . We also produce correlation plots for dispersion and anisotropy measures in Figure 5 and for the 2-step metrics in Figure 6. It can be seen that ODI is negatively correlated with FA and PA, and that IsoVF is positively correlated with MD and negatively with RTOP. 


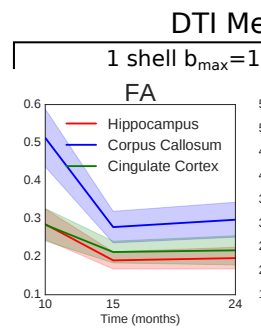

TI Metrics
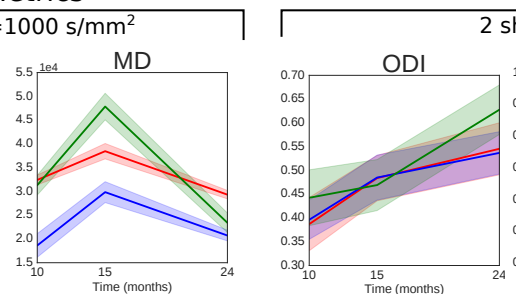

Time (months)
NODDI Metrics

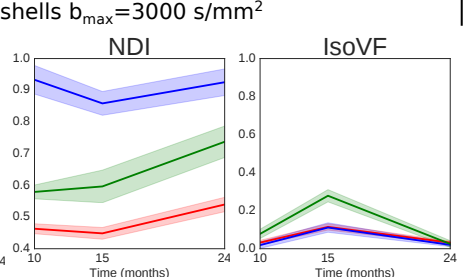

MAP-MRI Metrics

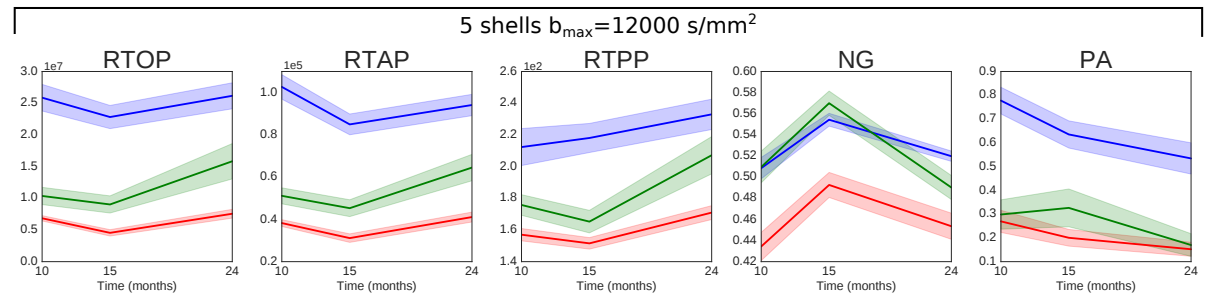

Fig. 4. DTI, NODDI and MAP-MRI metrics for the same time points in the hippocampus (red), corpus callosum (blue) and cingulate cortex (green).

\section{Discussion}

In this work, we have shown that different metrics of DTI, NODDI and MAPMRI appear to be sensitive to different processes as age-dependent cerebral amyloidosis manifests in both grey and white matter in the Alzheimer rats.

DTI findings: We find a significant drop in FA in all ROIs from 10 to 15 months and a small increase from 15 to 24 months. This corresponds with previous findings in the hippocampus using data up to $b=1000 \mathrm{~s} / \mathrm{mm}^{2}$ [8]. While a comparison of using different b-values in the DTI estimation was outside of the scope of this study, it was shown that when higher b-values are included, the FA trend consistently decreases over time [8]. Nonetheless, it has been argued that compared to FA, MD lends itself better to the assessment of cortical and subcortical grey matter, where net diffusion may not be expected to conform to any one specific direction [15]. When we assess MD, we consistently find an increase from 10 to 15 months and a decrease from 15 to 24 months. This may suggest that FA and MD are sensitive to different processes taking place in AD.

NODDI findings: Several studies have suggested that NODDI metrics, in particular ODI, have better AD classifying potential due to NODDI's ability to delineate signal contributions from different tissue compartments $[4,7]$. While we cannot do a classification study using our data, we find that ODI consistently increases in areas where tau pathology increases in our rat model [10]; the hippocampus, cingulate cortex and corpus callosum. We also find that IsoVF shows an increase from 10 to 15 months and a decrease from 15 to 24 months in all areas, following the same trend as DTI's MD. Though, it should be mentioned that fitting NODDI requires presetting the intra-cellular and isotropic diffusiv- 

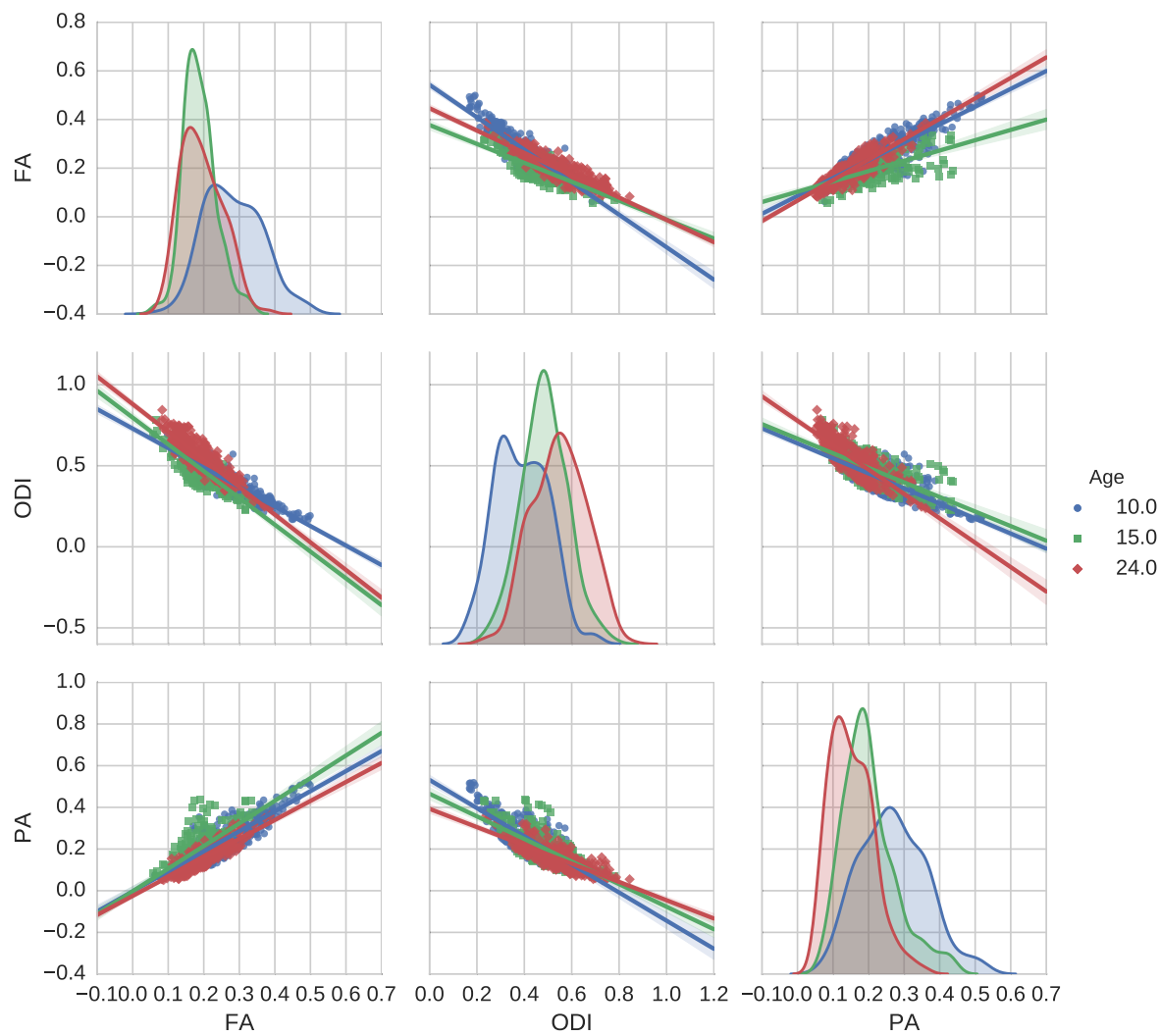

Fig. 5. Scatter plots of FA, ODI and PA for the rats of ages 10 months (blue), 15 months (green) and 24 months (red) in the hippocampus. It can be seen that ODI is negatively correlated with both FA and PA.

ity, which influences obtained metric values. Fitting NODDI on the selected $b_{\max }=3000 \mathrm{~s} / \mathrm{mm}^{2}$ or the full data does not significantly impact our findings.

MAP-MRI findings: To the best of our knowledge, this is the first study that estimates MAP-MRI metrics on data from an AD model. We find that all metrics except PA follow a two-stage progression pattern similar to DTI's MD. The decrease-increase of return-to-origin, return-to-axis and return-to-plane probability (RTOP, RTAP and RTPP) makes sense with the increase-decrease of MD, as an increased diffusivity means that spins are able to move away farther, reducing the chance they return to their origin, axis or plane. Interestingly, this does not make the signal more Gaussian, as the Non-Gaussianity follows an increasedecrease pattern in all ROIs. The exception to this trend is the RTPP in the corpus callosum, which increases monotonically, indicating a steady increase in restriction parallel to the axon direction. Finally, PA consistently decreases in all areas except the cortex, where a small increase is found, followed by a larger 

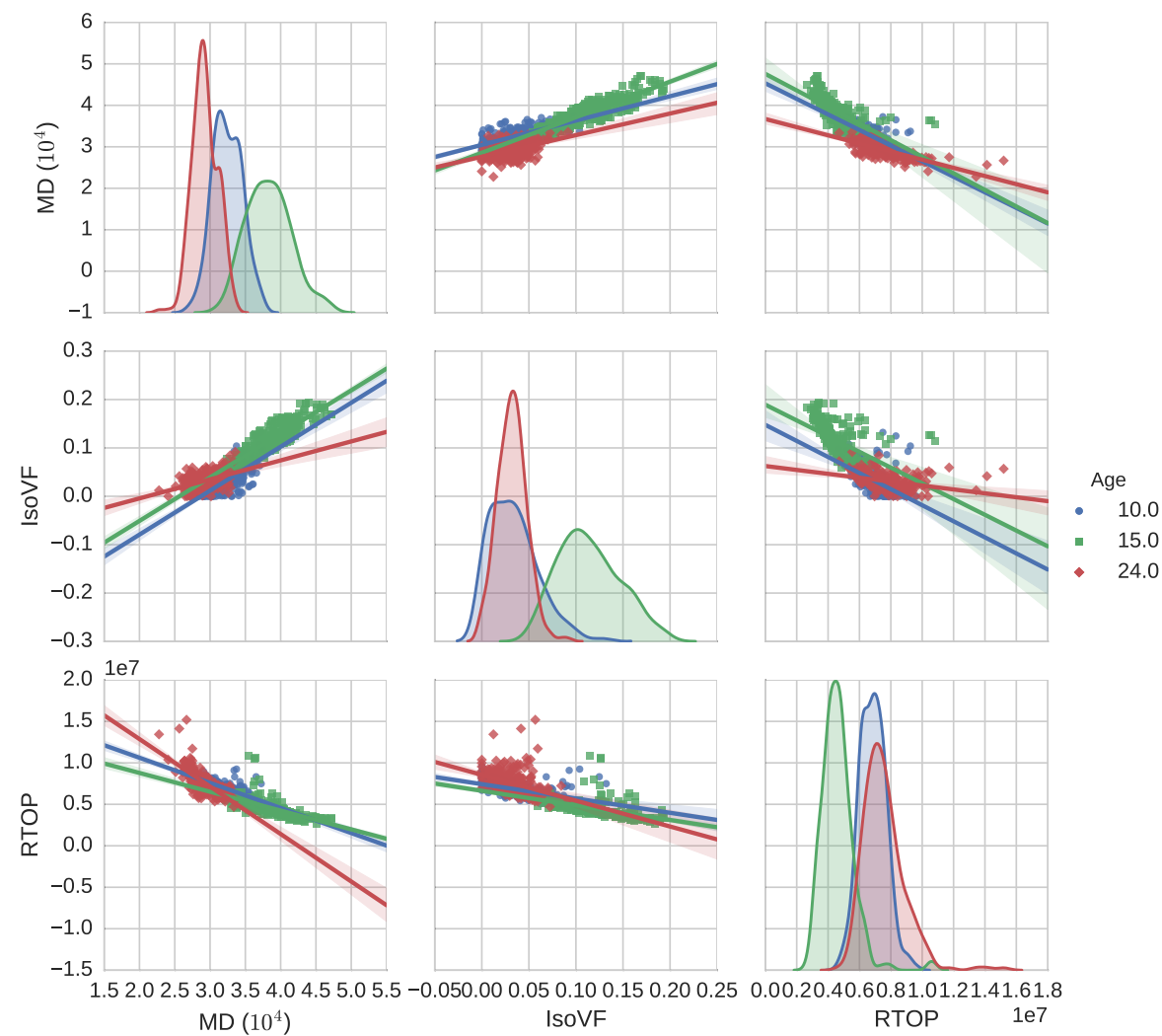

Fig. 6. Scatter plots of MD, IsoVF and RTOP for the rats of ages 10 months (blue), 15 months (green) and 24 months (red) in the hippocampus. It can be seen that IsoVF is positively correlated with MD and negatively with RTOP.

decrease. This decreasing trend in anisotropy measures when using higher gradients strengths was also reported with DTI's FA or HYDI's NQA [8]. We note that while we fitted MAP-MRI to the full data with 300 DWIs, it was shown that its metrics are stable under subsampling to less than 100 DWIs [14] or could even be fitted directly on a NODDI acquisition scheme.

Biological explanation for biomarker trends: The trends of all derived metrics can be divided into two groups: those that consistently decrease or increase and those that show a 'decrease-increase' or 'increase-decrease' pattern.

The first group could point towards the accelerating cerebral amyloidosis as age increases in these rats [10]. Over time, this "amyloid burden" results in agedependent neuronal demise that is likely owed to oligomeric $\mathrm{A} \beta$ accumulation. In turn, this neuronal demise could result in a more dispersed, less anisotropic diffusion signal. This corresponds with the observed correlations between dispersion and anisotropy measures in Figure 5. 
The second group may indicate an inflammatory response to amyloid accumulation, occurring prior to (or coincident with and obscuring) the onset of microstructural breakdown and macrostructural atrophy [16]. At 15 months TgF344-AD rats have heavy plaque burden and strong neuroinflammation, whereas by 24 months most of the inflammatory reaction to the plaques has passed. This corresponds to what we see when MD and IsoVF increase-decrease and RTOP, RTAP and RTPP decrease-increase (except RTPP at corpus callosum). The correlations between MD, IsoVF and RTOP in Figure 6 therefore makes sense. Though, the increase-decrease in NG indicates that while the inflammatory response increases diffusivity, it also increases the non-Gaussian portion of the signal at higher b-values.

Difficulties of comparing our findings with previous animal studies: There have been several previous dMRI studies using Alzheimer animal models. However, different species and disease expressions make comparisons of dMRI metrics difficult. For instance, our TgF344-AD rat model was made to drive cerebral amyloid and downstream tauopathy and neuronal loss, also known as the "amyloid cascade hypothesis" of John Hardy [17]. In contrast, the Tg4510 mouse model used by Colgan et al. [7] was developed to only assess tauopathy; and not the amyloid cascade hypothesis. For this reason, it is hard to make claims about differences in biomarker trends found between this study and theirs.

Limitations of the study: As we did not have healthy rats to statistically test for changes with disease progression - which means there is room for improvement - we used the youngest rat (10 months old) as a control subject to compare against suggestive changes at later time points. Another limitation is the low number of experimental subjects that also prevents us from statistically differentiating between the disease stages of the transgenic Alzheimer rat model.

\section{Conclusion}

We presented a unique study on transgenic Alzheimer rats at 10, 15 and 24 months, comparing DTI, NODDI and MAP-MRI-derived metrics, in grey and white matter areas known to manifest age-dependent cerebral amyloidosis that precedes neurofibrillary tangles and apoptotic loss of neurons. We found that NODDI's ODI and MAP-MRI's PA metrics uniformly changed over time, likely indicating that they are sensitive to age-dependent neuronal demise due to amyloid accumulation. It is relevant to note that both of these metrics require bvalues higher than $1000 \mathrm{~s} / \mathrm{mm}^{2}$. Conversely, we found that DTI's MD, NODDI's IsoVF and MAPMRI's RTOP, RTAP, RTPP and NG all follow a two-step progression from 10 to 15 to 24 months - either an increase-decrease or a decreaseincrease - likely indicating sensitivity to the neuroinflammatory response at 15 months and potentially, atrophy of the microstructure at 24 months. While this study does not have enough subjects to statistically differentiate between the different disease stages, it does provide valuable insight on which biomarkers and models come closest to explaining the biological changes in the cerebral tissue. 
Table 1. Mean and standard deviation of DTI, NODDI and MAP-MRI metrics for the three time points in each region of interest.

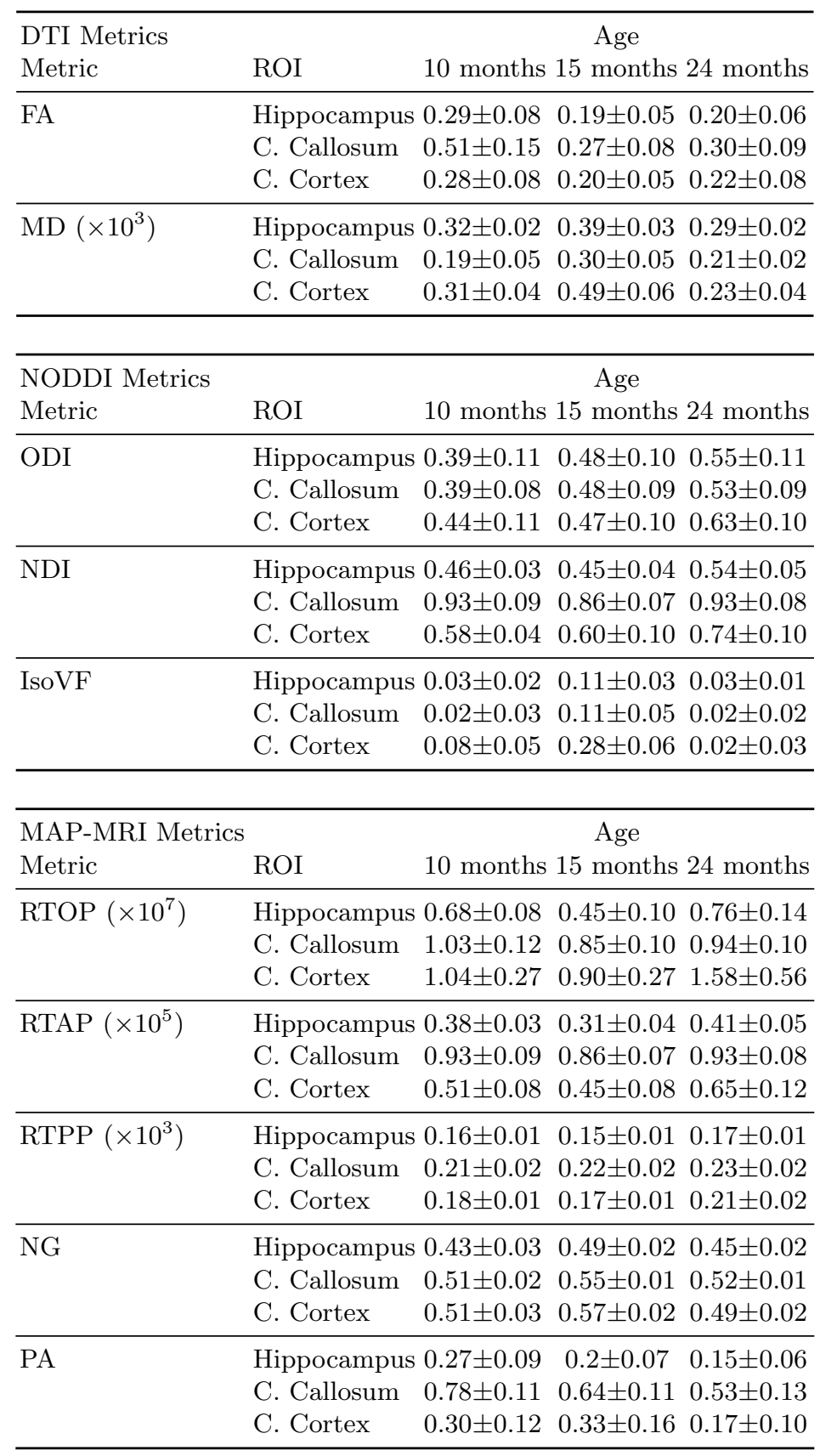




\section{Acknowledgments}

This work was partly supported by ANR "MOSIFAH" under ANR-13-MONU0009-01, the European Research Council (ERC) under the European Union's Horizon 2020 research and innovation program (ERC Advanced Grant agreement No 694665: CoBCoM) and NIH grants U54 EB020403 and R01NS076794.

\section{References}

1. Alzheimers Association. 2016 Alzheimers Disease Facts and Figures. Alzheimers \& Dementia 2016; 12(4).

2. Basser, Peter J., James Mattiello, and Denis LeBihan. "MR diffusion tensor spectroscopy and imaging." Biophysical journal 66.1 (1994): 259.

3. Zhang, Hui, et al. "NODDI: practical in vivo neurite orientation dispersion and density imaging of the human brain." Neuroimage 61.4 (2012): 1000-1016.

4. Nir, Talia M., et al. "Alzheimers disease classification with novel microstructural metrics from diffusion-weighted MRI." Computational Diffusion MRI. Springer International Publishing, 2016. 41-54.

5. Sahara, Naruhiko, et al. "Age-related decline in white matter integrity in a mouse model of tauopathy: an in vivo diffusion tensor magnetic resonance imaging study." Neurobiology of aging 35.6 (2014): 1364-1374.

6. Wells, J. A., et al. "In vivo imaging of tau pathology using multi-parametric quantitative MRI." Neuroimage 111 (2015): 369-378.

7. Colgan, N., et al. "Application of neurite orientation dispersion and density imaging (NODDI) to a tau pathology model of Alzheimer's disease." NeuroImage 125 (2016): 739-744.

8. Daianu, Madelaine, et al. "Multi-Shell Hybrid Diffusion Imaging (HYDI) at 7 Tesla in TgF344-AD Transgenic Alzheimer Rats." PloS one 10.12 (2015): e0145205

9. $\mathrm{Wu}, \mathrm{Yu}-\mathrm{Chien}$, and Andrew L. Alexander. "Hybrid diffusion imaging." NeuroImage 36.3 (2007): 617-629.

10. Cohen, Robert M., et al. "A transgenic Alzheimer rat with plaques, tau pathology, behavioral impairment, oligomeric a, and frank neuronal loss." The Journal of neuroscience 33.15 (2013): 6245-6256.

11. Özarslan, Evren, et al. "Mean apparent propagator (MAP) MRI: a novel diffusion imaging method for mapping tissue microstructure." NeuroImage 78 (2013): 16-32.

12. Garyfallidis, Eleftherios, et al. "Dipy, a library for the analysis of diffusion MRI data." Frontiers in neuroinformatics 8 (2014): 8.

13. Alexander, Daniel C., et al. "Orientationally invariant indices of axon diameter and density from diffusion MRI." Neuroimage 52.4 (2010): 1374-1389.

14. Fick, Rutger HJ, et al. "MAPL: Tissue microstructure estimation using Laplacianregularized MAP-MRI and its application to HCP data." NeuroImage 134 (2016): 365-385.

15. Chiapponi, Chiara, et al. "Cortical grey matter and subcortical white matter brain microstructural changes in schizophrenia are localised and age independent: a casecontrol diffusion tensor imaging study." PloS one 8.10 (2013): e75115.

16. Weston, Philip SJ, et al. "Diffusion imaging changes in grey matter in Alzheimers disease: a potential marker of early neurodegeneration." Alzheimer's research \& therapy 7.1 (2015): 1-8.

17. Hardy, John A., and Gerald A. Higgins. "Alzheimer's disease: the amyloid cascade hypothesis." Science 256.5054 (1992): 184. 